\title{
Chapter 11 \\ Integration of Building Information \\ Modelling and Augmented Reality \\ for Building Energy Systems \\ Visualisation
}

\section{Vishak Dudhee and Vladimir Vukovic}

\begin{abstract}
Buildings consist of numerous energy systems, including heating, ventilation, and air conditioning (HVAC) systems and lighting systems. Typically, such systems are not fully visible in operational building environments, as some elements remain built into the walls, or hidden behind false ceilings. Fully visualising energy systems in buildings has the potential to improve understanding of the systems' performance and enhance maintenance processes. For such purposes, this paper describes the process of integrating Building Information Modelling (BIM) models with Augmented Reality (AR) and identifies the current limitations associated with the visualisation of building energy systems in AR using BIM. Testing of the concept included creating and superimposing a BIM model of a room in its actual physical environment and performing a walk-in analysis. The experimentation concluded that the concept could result in effective visualisation of energy systems with further development on the establishment of near real-time information.
\end{abstract}

Keywords Augmented reality • BIM • Buildings $\cdot$ Energy systems • Visualisation

\subsection{Introduction}

The buildings and construction sector accounted for 36\% of final energy use and $39 \%$ of energy and process-related carbon dioxide (CO2) emissions in 2018 [1]. In order to meet sustainability goals and mitigate climate change, energy systems must be more effective [2]. The visualisation of energy systems' performance can enhance the user's understanding of the system and, therefore, their decision-making relating to them [3]. Buildings consist of several energy systems, such as the heating, ventilation, and air conditioning (HVAC) systems, lighting systems and electric motors, out of which heating, space cooling, water heating, and lighting accounted for nearly $70 \%$ of site energy consumption [4]. The integration of Building Information Modelling

\footnotetext{
V. Dudhee $(\varangle) \cdot$ V. Vukovic

School of Computing, Engineering and Digital Technologies, Teesside University, Middlesbrough, UK

e-mail: V.Dudhee@tees.ac.uk
} 
(BIM) and Augmented Reality (AR) provides the construction industry with many benefits, such as checking building designs [5, 6]. Although BIM models contain the energy systems information, the possibilities for visualising and analysing building energy system performance and behaviour by integrating BIM and AR have not been sufficiently explored.

\subsection{Method}

To explore the possibility of visualising digital data of energy systems and to understand the current limitations associated with the usage of BIM and AR for energy system visualisation, a BIM model was generated and visualised in AR using the process illustrated in Fig. 11.1.

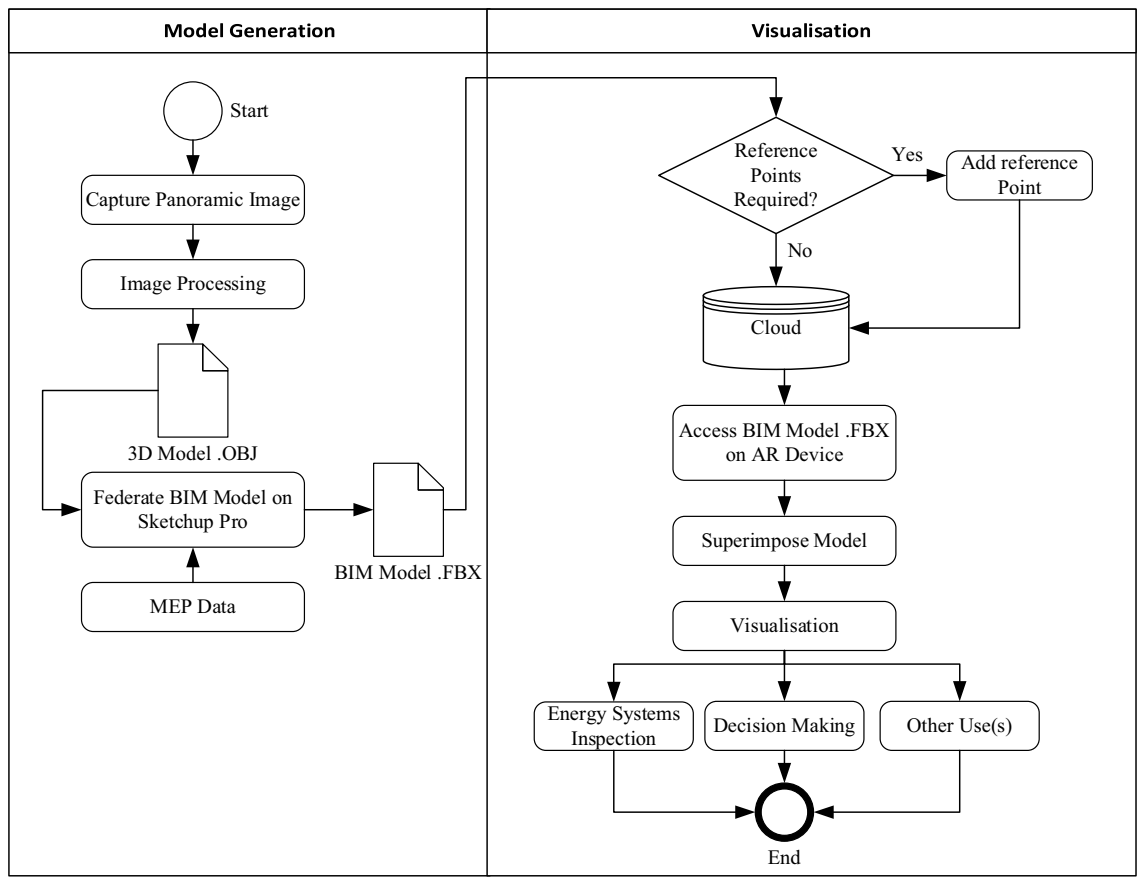

Fig. 11.1 BIM modelling and visualisation in AR schematic flowchart 


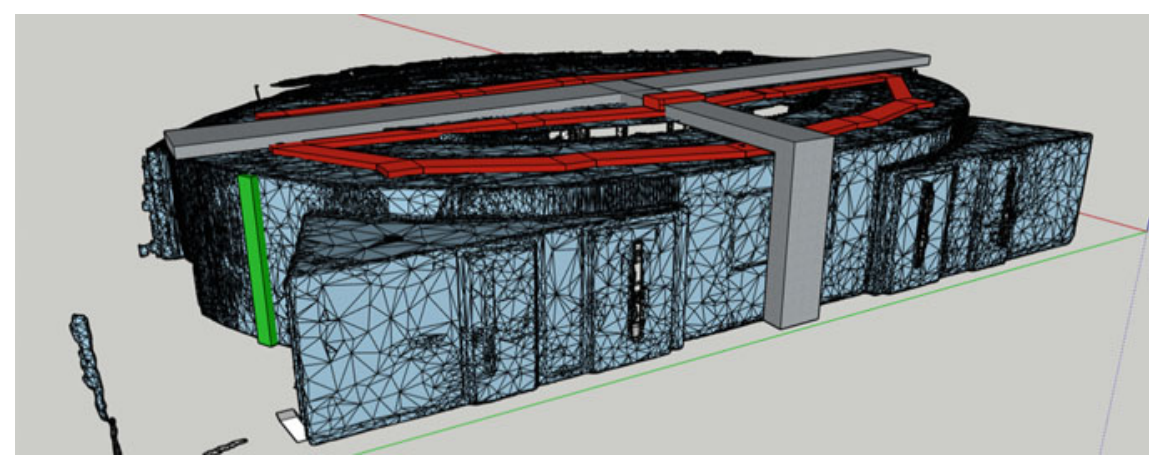

Fig. 11.2 Room mesh model with MEP components

\subsubsection{Generation of a BIM Model}

For the purposes of experimentation, a BIM model of a room at Teesside University with mechanical, electrical and plumbing (MEP) data was created, as shown in Fig. 11.2. An oval-shaped room was selected as atypical and particularly challenging for the traditional computer aided design software [7]. Therefore, the 3D model of the selected room was first automatically generated using a 3D camera (Matterport Pro 2) and Matterport app. SketchUp Pro was used to design and add MEP data and information to the model. The 3D model of the meeting room in OBJ format was imported and scaled 1:1 to avoid any issues that could arise related to scaling. A layer was then added for the room model, and MEP data were drawn and textured using three different layers and colours. The electrical lighting components are represented in red, the HVAC systems in grey and the piping system in green. Textual information was added near appropriate elements of the building to give further technical details of the systems.

\subsubsection{Visualisation in AR}

The generated model of the room was superimposed to the actual physical building using freely available software 3D Viewer Beta and visualised in Augmented Reality equipment Microsoft HoloLens 1-Developers Edition. The AR device's spatial mapping system generates a map of meshes that represent the physical space, which enables the placement of the digital model into the physical environment [8]. Using the 3D Viewer Beta AR software, the model was superimposed by trial and error. Since the software does not have a prescribed way of superimposing objects, the model was superimposed using arbitrary reference points. The appearance of physical systems found in the building was analysed to understand the possible uses of $\mathrm{AR}$ and current limitations in visualising energy systems. 


\subsection{Results}

The room was visualised in AR using two different approaches: with and without the MEP systems added to the model. It was possible to successfully open the model using both approaches on AR software and interact with such models in the AR environment. The interaction and response times of the model were slow due to the large number of mesh elements it needed to process with the curved shape of the room. The different components of the room, such as the doors, windows and room furniture were visible; therefore, the different sections, such as the walls and ceiling, could be recognised. Although the model's texture was imported from SketchUp which showed colouring, the colour visualised in AR was plain white. There was a clear quality difference between the model in OBJ format and the model in the AR environment in FBX format. However, the model did show the actual geometry of the components, as presented in Fig. 11.3.

The MEP data were visible, and the system's components appeared to be on top of the ceiling. The electrical component added to the model logically matched the room lighting. The mechanical ventilation system was also positioned in a way so that it met the room ventilation cap and made its way out, passing through the ceiling and wall cavity. The water pipe, added as the plumbing system of the building, was also visible and located at the correct position. The written information about the different MEP systems and room components was visible and readable. Although the colour was not as assigned and presented in Fig. 11.2, the geometry was visible as modelled and positioned.

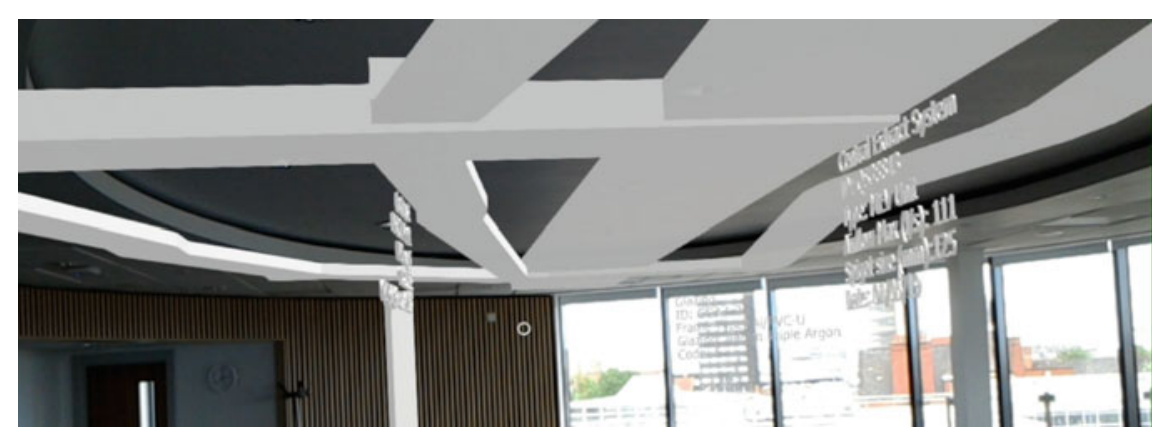

Fig. 11.3 Model visualisation in AR environment 


\subsection{Discussion}

\subsubsection{Visualisation Procedure}

The visualisation procedure depends on various factors, such as the software used to create the BIM model, type of AR equipment and AR software being used. For this experiment, the BIM model created as described in Fig. 11.1 consisted of limited information, whereas having a more detailed BIM model could provide a better insight. The type of AR equipment used also had a significant impact on the AR experience. Mixed Reality (MR) headsets move with the user's head and do not limit the user from using their hands. In contrast, handheld AR equipment must be adequately positioned with the user's hands, potentially obstructing any simultaneous maintenance activities. The range of options available when interacting with BIM in an AR environment depends on the AR software being used. As a freely available software with limited features was used in the current study, it was not possible to view the texture or colour of the components in the model. Using this technique can be time-consuming and requires effort to achieve any level of accuracy in superimposing the model. This technique is not always feasible and can introduce alignment errors between the model and physical systems. Because the rotation and movement of the model in AR environment are completed by manipulating a sliding scale without defined values rather than with exact ratios, inaccuracies are common. For the model to be superimposed onto the physical building, the model needs to be aligned both horizontally and vertically using reference points and positioned on a horizontal flat surface before scaling can facilitate the superimposing process.

\subsubsection{Current Limitations}

A walk-through analysis of the model revealed that when the user moves through the space, the device loads only a particular section of the model within the user's field of view. Due to this loading process, inaccuracies in placement of model components were noticed during walk-throughs. For example, Fig. 11.4 shows the shifted position of the vertical pipes when viewed from different viewpoints in the room. Such imprecise positioning can result in the misinterpretation of building information.

Additional limitation is related to the type of data that could be viewed in AR, constrained to the geometrical design and pre-set information of the energy systems. The behaviour and performance of the system could not be identified or analysed in AR using the described approach. The presented approach is limited to visualisation of the static information of energy systems found in the BIM model. Not having the ability to visualise near real-time system information and the surrounding environmental information limits the possible uses of such visualisation systems. With 


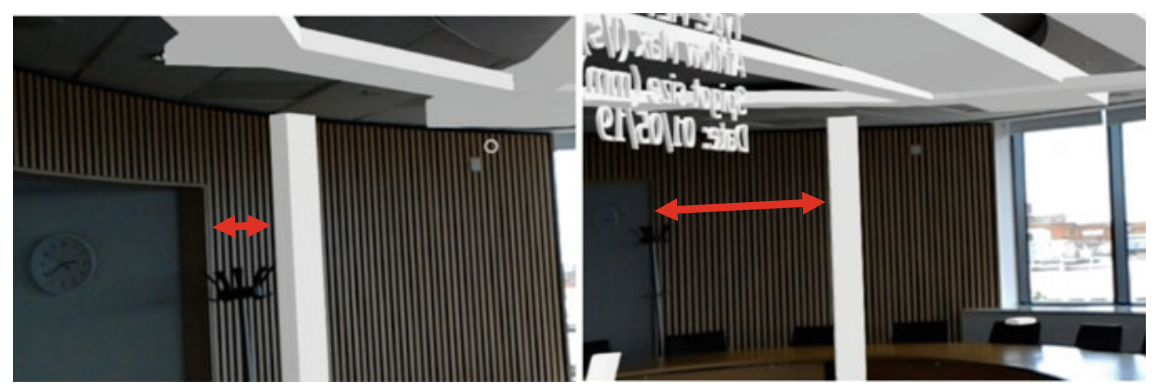

Fig. 11.4 Floating graphics

commercial usage of AR currently being under development, a limited variety of BIM-AR software is available, and none were identified as specialising in building energy system visualisation.

\subsection{Conclusion}

BIM models integrated with AR can be used to effectively visualise energy systems in a building, provided that the model contains the necessary and relevant information. The use of such a visualisation framework can allow for the analysis of building systems and the collection of necessary information found in the BIM model. BIM and AR's possible usage is still limited due to lack of real-time information and imprecise positioning during walk-throughs, which will be the subject of future work. The inclusion of near real-time data in BIM-AR visualisation techniques may improve understanding of system operation. Such integration framework will be explored in the future.

Acknowledgements The authors would like to acknowledge the help and financial support of Dr Kevin Thomas, Associate Dean International, School of Science, Engineering and Design, Teesside University who facilitated acquisition of Matterport services used in this study.

\section{References}

1. International Energy Agency, 2019 Global Status Report for Buildings and Construction. COP25. (UN Environment and the International Energy Agency, Spain, 2019)

2. H. Alemasoom, F. Samavati, J. Brosz, D. Layzell, EnergyViz: an interactive system for visualisation of energy systems. Vis. Comput. 32, 403-413 (2016)

3. M.R. Herrmann, D.P. Brumby, T. Oreszczyn, X.M. Gilbert, Does data visualization affect users' understanding of electricity consumption? Build. Res. Inf. 46, 238-250 (2018)

4. V.S. Harish, A. Kumar, A review on modeling and simulation of building energy systems. Renew. Sustain. Energy Rev. 56, 1272-1292 (2016) 
5. X. Wang, M. Truijens, L. Hou, Y. Wang, Y. Zhou, Integrating augmented reality with building information modeling: onsite construction process controlling for liquefied natural gas industry. Autom. Constr. 40, 96-105 (2014)

6. C. Chai, K. Mustafa, S. Kuppusamy, A. Yusof, C.S. Lim, S.H. Wai, BIM integration in augmented reality model. Int. J. Technol. 10, 1266-1275 (2019)

7. N. Kocic, Creating 3D model of architectural objects in different software. J. Ind. Des. Eng. Graph. 14, 249-252 (2014)

8. G. Evans, J. Miller, M.I. Pena, A. MacAllister, E. Winer, Evaluating the Microsoft HoloLens through an augmented reality assembly application, in Mechanical Engineering Conference Presentations, Papers, and Proceedings 10197:0V1-0V16 (2017)

Open Access This chapter is licensed under the terms of the Creative Commons Attribution 4.0 International License (http://creativecommons.org/licenses/by/4.0/), which permits use, sharing, adaptation, distribution and reproduction in any medium or format, as long as you give appropriate credit to the original author(s) and the source, provide a link to the Creative Commons license and indicate if changes were made.

The images or other third party material in this chapter are included in the chapter's Creative Commons license, unless indicated otherwise in a credit line to the material. If material is not included in the chapter's Creative Commons license and your intended use is not permitted by statutory regulation or exceeds the permitted use, you will need to obtain permission directly from the copyright holder.

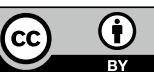

